\title{
Promoción del lavado de manos en niños de 10 años: evaluación de intervenciones piloto en ciudades del Norte
}

\section{Vol. 11, №3, 33-45}

revistas.unc.edu.ar/inde x.php/racc

\section{Argentino}

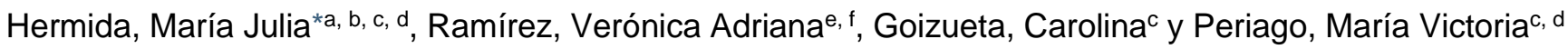

\section{Artículo Original}

\section{Resumen}

Aunque el lavado de manos evita enfermedades infecciosas graves a bajo costo, sólo una de cada cinco personas se lava las manos después de ir al baño. Este estudio presenta una intervención diseñada interdisciplinariamente, corta y económica para promover el conocimiento sobre el lavado de manos en niños de 10 años y dos estudios piloto para evaluar su impacto. En el piloto 2016 (Puerto Iguazú, Argentina), se compararon las respuestas a una encuesta sobre lavado de niños que participaron y no participaron de la intervención. La intervención consistió en actividades lúdicas sobre el lavado de manos. En el piloto 2017 (cuatro localidades del norte argentino), se compararon las respuestas de niños previas y posteriores a la intervención. Los resultados de ambos estudios piloto indicaron que esta intervención tiene potencial para promover el conocimiento sobre los pasos que deben seguirse para un correcto lavado de manos.

\section{Abstract}

Handwashing promotion in 10-year-old children: intervention studies in Northern Argentina Although handwashing prevents serious infectious diseases at low cost, only one in five people wash their hands after using the bathroom. This study presents an interdisciplinary, short-term and low-cost intervention aimed at promoting knowledge about handwashing in 10-year-old children and two pilot studies to assess its impact. In the 2016 pilot study, conducted in Puerto Iguazú city, the responses to a survey about handwashing of children who participated and did not participate in the intervention were compared. The intervention consisted in playful activities in which children were instructed in different aspects of handwashing. In the 2017 pilot, carried out in four locations in northern Argentina, the responses of children before and after the intervention were compared. The results of both pilot studies indicated that this intervention has the potential to promote knowledge about the steps needed to achieve proper hand washing.
Palabras clave:

lavado de manos, conocimiento, niños,

intervenciones.

Recibido el 3 de noviembre de 2015; Aceptado el 25 de agosto de 2019

Editaron este artículo: Giselle Kamenetzky, Paula Abate, Sebastian Miranda, María Victoria Ortiz y Pablo Correa

\section{Introducción}

Una de las estrategias de salud pública más eficaces y económicas es el lavado de manos con agua y jabón. Se trata de un hábito que permite prevenir enfermedades graves a muy bajo costo
(Larson, 1988). Diferentes estudios han demostrado que el lavado de manos reduce las muertes causadas por diarrea a casi la mitad (Curtis \& Cairncross, 2003) y las infecciones

\footnotetext{
a Universidad Nacional de Hurlingham, Instituto de Educación, Buenos Aires, Argentina.

b Universidad Torcuato Di Tella, Buenos Aires, Argentina.

${ }^{c}$ Fundación Mundo Sano, Buenos Aires, Argentina.

${ }^{d}$ Consejo Nacional de Investigaciones Científicas y Técnicas, Buenos Aires, Argentina.

e Unidad de Neurobiología Aplicada (UNA, CEMIC-CONICET), Buenos Aires, Argentina.

f Universidad de Buenos Aires, Facultad de Psicología, Buenos Aires, Argentina.

"Enviar correspondencia a: Hermida, J. E-mail: julia.hermida@gmail.com
}

Citar este artículo como: Hermida, M. J., Ramírez, V. A., Goizueta, C. \& Periago, M. V. (2019). Promoción del lavado de manos en niños de 10 años: evaluación de intervenciones piloto en ciudades del Norte Argentino. Revista Argentina de Ciencias del Comportamiento, 11(3), 33-45 
Hermida, M. J., Ramírez, V. A., Goizueta, C. y Periago, M. V./ RACC, 2019, Vol. 11, №3, 33-45

agudas respiratorias a un cuarto (Rabie \& Curtis, 2006). Por ejemplo, el lavado de manos con jabón antes de comer y después de ir al baño reduce la infección por parásitos, incluyendo los geohelmintos (Garn et al., 2016; Grimes et al., 2016; Wu et al., 2016). Otros estudios muestran que podría reducir la incidencia de diarrea en un 40\% (Ejemot-Nwadiaro, Ehiri, Arikpo, Meremikwu, \& Critchley, 2015). Considerando que la diarrea implica 1.8 millones de muertes anuales (Walker et al., 2013), especialmente entre niños pobres (Woldemicael, 2001), sería esperable que el lavado de manos sea un hábito cotidiano para toda la población.

Sin embargo, a nivel mundial, sólo una de cada cinco personas aproximadamente se lava las manos después de ir al baño (Freeman et al., 2014). Si bien los factores que afectan la práctica del lavado de manos son complejos (Whitby et al., 2007), la evidencia indica que tanto la disponibilidad de agua y jabón, como el conocimiento, son factores cruciales en la adquisición del hábito (Naikoba \& Hayward, 2001). En este sentido, aumentar el conocimiento sobre la importancia del lavado de manos y sobre cómo realizarlo adecuadamente podría contribuir a generar el hábito. Si bien algunos estudios han demostrado la existencia de una brecha entre el conocimiento sobre el lavado de manos y la acción de lavarse en niños en edad escolar (Demberere, Chidziya, Ncozana, \& Manyeruke, 2016; Rabbi \& Dey, 2013; Tamilarasi, Arunmozhi, Raja \& Rajajeyakumar, 2016), el conocimiento sobre higiene es considerado un punto de partida para la potencial adquisición de este hábito. Desde la teoría cognitiva social, Bandura (2004) especifica un conjunto de determinantes para la adquisición de hábitos saludables. Estos determinantes principales incluyen, entre otros, el conocimiento de los beneficios de las prácticas de salud, la autoeficacia percibida (percepción de que uno puede ejercer control sobre sus hábitos de salud), las expectativas de resultados en comparación con los costos, y los facilitadores e impedimentos sociales y estructurales de los cambios que buscan (Bandura, 1977, 2004). Adquirir hábitos saludables implica esfuerzo. Contar con la información sobre las prácticas saludables crea la condición previa para realizarlo. Si las personas carecen del conocimiento sobre cómo sus hábitos de vida afectan su salud y cómo podrían cambiarlos, tienen pocas razones para iniciar los esfuerzos de modificar sus hábitos perjudiciales (Bandura, 2004; McAlister, Perry, \& Parcel, 2008). Entonces, aunque con el conocimiento no basta para adquirir hábitos saludables, es una precondición necesaria para que tales cambios se produzcan (Fisher \& Fisher, 1992).

Muchas veces los hábitos están enraizados en las familias y por eso son difíciles de modificar (Borra, Kelly, Shirreffs, Neville, \& Geiger, 2003). Por ello, las escuelas, como lugar ajeno a las familias, tienen un importante potencial en la promoción de cambio de hábitos (Bandura, 2004). En particular, algunas investigaciones sugieren que los niños son buenos agentes de cambio (Mitchell, Fraser, \& Bearon, 2007; Percy-Smith \& Burns, 2013), incluyendo en casos de obesidad infantil con cambio de hábitos alimenticios en los niños y por ende en sus padres (Basdevant, Boute, \& Borys, 1999), en casos de desarrollo comunitario (PercySmith \& Burns, 2013) y en el cuidado del medio ambiente (Heft \& Chawla, 2006; Mitchell, Ling, Krusekopf, \& Kerr, 2015). Estos resultados sugieren que los niños podrían constituirse en factores promotores del lavado de manos.

Ante este panorama, es crucial diseñar y poner a prueba diferentes intervenciones tendientes a mejorar el conocimiento acerca del lavado de manos. Esto se torna especialmente importante en poblaciones de bajos recursos, con falta de servicios básicos y con poco acceso al sistema de salud, ya que estas poblaciones son las más vulnerables a las enfermedades por diferentes agentes que causan infecciones respiratorias, diarrea y otros trastornos intestinales. Por ejemplo, la prevalencia de parásitos intestinales varía según el estatus socioeconómico, las condiciones ambientales y sanitarias y el acceso al agua (Dib, Oquilla, Lazarte, \& Gonzalez, 2012; Freeman et al., 2015; Gamboa et al., 2011; Zonta, Navone, \& Oyhenart, 2007), debido a que el principal modo de transmisión de los agentes etiológicos es a través de la contaminación fecal del suelo y los alimentos, así como del agua utilizada para beber y cocinar. Además, tal como muestran estudios locales (Gamboa et al., 1996; Milano, Oscherov, Palladino, \& Bar, 2007) y foráneos (Cairncross, Bartram, Cumming \& Brocklehurst, 2010; Chin et al., 2016; Mahmud et al., 2015; Manz et al., 2017; Steinmann 
Hermida, M. J., Ramírez, V. A., Goizueta, C. y Periago, M. V./ RACC, 2019, Vol. 11, №3, 33-45

et al., 2007), el hacinamiento, malnutrición, falta de hábitos de higiene y el manejo y tratamiento de residuos también son factores influyentes en la transmisión.

Diversas intervenciones se han puesto en marcha para promover el lavado de manos a nivel mundial (Freeman et al., 2014; Naikoba \& Hayward, 2001). En Argentina, tres estudios muestran que intervenciones basadas en la educación, la práctica y la provisión de elementos para el lavado mejoraron la adquisición de este hábito en personal hospitalario (Rodríguez et al., 2015; Rosenthal, Guzman, \& Safdar, 2005) y pacientes (Rosenthal, Guzman \& Safdar, 2004). Sin embargo, todas estas intervenciones han involucrado a población adulta y urbana o periurbana. Hasta nuestro conocimiento no se han reportado intervenciones para promover el lavado de manos en niños; menos aún, en niños de localidades no-urbanas de bajo nivel económico social.

El presente trabajo describe una intervención diseñada para promover el conocimiento sobre lavado de manos en niños de 10 años de edad de localidades del Norte Argentino. La intervención consistió en un evento (jornada de juego) con los niños en ocasión del Día Mundial del Lavado de Manos (The Global Handwashing Partnership, 2017), destinada a mejorar el conocimiento sobre el lavado de manos. El presente informe analiza dos estudios piloto sobre el impacto del evento y sugiere líneas futuras de investigación.

\section{Método}

\section{Participantes}

Se seleccionaron niños de $4^{\circ}$ grado para asegurar que la herramienta de evaluación y las actividades fueran comprendidas ya que precisaban de lectura y escritura, habilidades que se adquieren a lo largo del primer ciclo educativo ( $1^{\circ}$ a $3^{\circ}$ grado). Los niños participantes pertenecían a escuelas públicas de localidades no-urbanas con menos de 85.000 habitantes en provincias con altos índices de pobreza (Instituto Nacional de Estadísticas y Censos [INDEC], 2010).

El primer Piloto se realizó en la ciudad de Puerto Iguazú (Provincia de Misiones, Argentina) en octubre de 2016. El segundo piloto se realizó en octubre de 2017 en las localidades de Tartagal (Provincia de Salta, Argentina), Clorinda (Provincia de Formosa, Argentina), Pampa del Indio
(Provincia de Chaco, Argentina) y Puerto Iguazú.

En el Piloto 2016, participaron del evento 400 niños de seis escuelas. Sin embargo, de la evaluación post-intervención sólo participaron 172 niños (de cuatro escuelas), 74 que asistieron al evento y 98 que no asistieron. La edad promedio de los niños que respondieron la encuesta fue 10 $(D E=.95)$ años.

En el segundo estudio piloto (2017), 748 niños de 23 escuelas participaron del evento. El número total de encuestas obtenido fue de 709 para la evaluación pre-intervención (T1 de aquí en adelante) y 584 para la evaluación postintervención (T2 de aquí en adelante). Dichas encuestas correspondieron a 17 escuelas de cuatro localidades. La edad promedio de los niños fue $9.45(D E=1.05)$ años en T1 y $9.54(D E=.99)$ años en T2.

\section{Diseño}

Para la evaluación del piloto 2016, se realizó una evaluación posterior al evento que comparaba el conocimiento sobre el lavado de manos de niños que habían asistido al evento con el de niños que no habían asistido, un mes después del evento. Para la evaluación del piloto 2017, se propuso un diseño pre - post, en el cual los niños fueron evaluados en cuanto a su conocimiento del lavado de manos un mes antes (T1) y un mes después del evento (T2).

Para asegurar la comparabilidad de las actividades en las distintas localidades, coordinadores residentes en cada localidad fueron capacitados en las actividades y los contenidos de cada actividad: pasos para el lavado de manos, momentos en los que hay que lavarse las manos, razones para el lavado de manos y elementos para el lavado. Además, los coordinadores requirieron la participación de un mínimo de tres ayudantes más, también residentes de cada localidad, para el desarrollo de la logística del evento y su evaluación.

Diseño de la intervención. El diseño de las actividades del evento se realizó con la participación de profesionales de distintas disciplinas: especialistas en diagnóstico y prevención de transmisión de parásitos y especialistas en aprendizaje infantil. Además, una profesora nacional de educación física colaboró en el diseño de la dinámica de los juegos y en asegurar el formato lúdico de los mismos y un 
Hermida, M. J., Ramírez, V. A., Goizueta, C. y Periago, M. V./ RACC, 2019, Vol. 11, №3, 33-45

diseñador colaboró en el diseño de los materiales necesarios para las actividades del evento.

Para el diseño de las actividades se siguieron los siguientes pasos:

1) Se seleccionaron cuatro contenidos sobre el lavado de manos para ser trabajados en el evento, en función a los contenidos sugeridos para la promoción del lavado en el Día Mundial del Lavado de Manos (Global Handwashing Partnership, 2017):

a) Pasos para lavarse las manos (en adelante "Pasos"), es decir, cómo se realiza un correcto lavado de manos, qué pasos y en qué orden deben seguirse. Esto incluye cierto tipo de información que habitualmente es desconocida, como por ejemplo la necesidad de frotarse las manos durante al menos 20 segundos para la eliminación de agentes transmisores (Boyce \& Pittet, 2002).

b) Momentos en que es necesario lavarse las manos (en adelante "Momentos"), es decir, cuándo debemos lavarnos para prevenir el contagio de enfermedades. Esto incluye dos momentos fundamentales: antes de comer o manipular alimentos y después de ir al baño (Larson \& APIC Guidelines Committee, 1995).

c) Razones para lavarse las manos (en adelante "Razones"), es decir, por qué es importante lavarse. Esto incluye brindar información acerca de la transmisión de gérmenes que podrían causar enfermedades graves como la diarrea. También implica informar que estos microorganismos se transmiten aunque no sean visibles, por lo que hay que lavar las manos aunque parezca a la vista que están limpias (Larson, 1988).

d) Elementos necesarios para lavarse las manos (en adelante "Elementos"), es decir, con qué debemos lavarnos. Esto incluye informar acerca de la necesidad del jabón o de alguna sustancia que permita el arrastre de microorganismos. El jabón puede ser reemplazado por otras sustancias como arena o ceniza. Además, implica informar que el lavado debe hacerse indefectiblemente con agua corriente ya que sumergir las manos en agua contaminada previamente con microorganismos implica la posibilidad de la transmisión de gérmenes mediante el agua (Pittet, Allegranzi, Boyce, \& World Health Organization World Alliance for Patient Safety First Global Patient Safety Challenge Core Group of Experts, 2009).

2) A continuación se diseñaron actividades para trabajar los contenidos seleccionados. Las actividades tenían las siguientes características:

a) Fueron trabajadas previamente con las docentes de cada sala invitada a participar del evento, es decir, recibieron un documento con el detalle de las actividades y, días antes del evento, tuvieron una reunión con los asistentes de los investigadores a fin de despejar dudas. Dichas docentes fueron invitadas a llevar las actividades a cabo en conjunto con colaboradores de la investigación, para que ellas puedan luego replicarlas por fuera del evento si lo deseaban. Estudios previos que involucran docentes de grado y de educación física en la aplicación de intervenciones en salud han mostrado resultados positivos (Gortmaker et al., 1999).

b) Cada juego tenía una introducción, un nudo (lúdico) y un cierre. En estudios educativos locales y foráneos se ha mostrado que esta forma de trabajo, que implica planificar estrategias de resolución y luego evaluar la ejecución, favorece el aprendizaje (Diamond, 2013).

c) Las actividades fueron pensadas para niños de cuarto grado; sin embargo, algunas de ellas tienen la posibilidad de adaptación a diferentes niveles de dificultad, es decir, que las actividades podían hacerse más fáciles o más difíciles según el grupo de niños. El modo de aumentar o disminuir la dificultad estaba sistematizado siguiendo criterios similares a los utilizados en estudios locales previos (Hermida et al., 2015).

d) Todas tenían formato lúdico y poca duración (20 minutos máximo), para favorecer la concentración y motivación de los niños (Betts, Mckay, Maruff, \& Anderson, 2006).

Para el piloto 2016 se diseñaron cuatro actividades, una para cada contenido: Pasos, Momentos, Razones y Elementos, y se pusieron a prueba. Para que los niños puedan rotar entre las actividades, era necesario que todas ellas tuvieran la misma duración, pero dos de las cuatro actividades (las correspondientes a Razones y Momentos) resultaron 10 minutos promedio más breves que las otras, lo que producía que las salas asignadas a estas actividades más breves tuvieran que esperar a que finalice la actividad siguiente. Por lo tanto, el evento de 2017 resultó en tres actividades que harían foco en los siguientes contenidos: Elementos, Razones y Momentos como una actividad conjunta y finalmente Pasos 
Hermida, M. J., Ramírez, V. A., Goizueta, C. y Periago, M. V./ RACC, 2019, Vol. 11, N3, 33-45

para el lavado de manos. Las actividades se describen en la Figura 1.

3) Se pensaron los materiales necesarios, cómo conseguirlos y trasladarlos hasta las localidades, o bien cómo producirlos en cada localidad.

4) Se revisaron los materiales para tratar de generar opciones de bajo costo.

5) Se planificó la dinámica de circulación de los niños por los juegos. Las tres actividades se realizaban en simultáneo y cada sala de niños rotaba por las tres actividades.

6) Se diseñó un material escrito para explicar las actividades que pueda ser comprendido por docentes de las escuelas. Las actividades fueron explicadas en una reunión a colaboradores locales,

\section{Búsqueda del tesoro}

Cada sala es un equipo que cuenta con una pista inicial a partir de la cual deberá ir buscando 5 sobres por la plaza. Cada sobre contiene una imagen de un paso del lavado de manos y una pista de dónde buscar el siguiente sobre. Al encontrar el último sobre, cada equipo ordena las 5 imágenes en un afiche, Un representante explica al resto de las salas los pasos del lavado de manos.

\footnotetext{
Carrera de postas

Cada equipo forma una fila con un niño cada $20 \mathrm{mts}$. Al inicio de cada fila hay un canasto (con 10 productos de cocina o baño espolvoreados con brillantina), y otro vacio al final. El primer niño de la fila toma un producto y se lo lleva corriendo al segundo. El producto se pasa de niño en niño hasta que el último lo coloca en el canasto final. El primer nif⿵o toma un nuevo objeto del canasto inicial y repite la operación. Gana el equipo que traslada antes todos los elementos. Luego se reflexiona sobre si hay que lavarse las manos antes o después de manipular productos de baño o de cocina.
}

quienes estuvieron a cargo de organizarlas en cada localidad. Dichos colaboradores trabajaron conjuntamente con las escuelas para obtener las autorizaciones de los padres para responder las encuestas y asistir al evento. También ellos interactuaron con las docentes en forma previa al evento para comentarles las actividades y solicitarles su participación durante el evento.

7) Se organizó la logística del evento. En octubre de 2017, cada evento se realizó en el Polideportivo, predio municipal de cada localidad o en cada escuela. Todos los eventos tuvieron entre 3 y 4 horas de duración. Al finalizar el evento se entregaron a los niños folletos, calcomanías y pulseras, como recordatorio de los contenidos trabajados

\section{Objetivo}

Asimilar cuáles son las razones por las que es importante el lavado y los momentos en que es imprescindible lavarse las manos

\section{Materiales}

Cajas de cartón, productos de baños o cocina, brillantina

\section{Objetivo}

Conocer los pasos para un correcto lavado de manos

\section{Materiales}

Sobres, papel es con pistas escritas, afiche, plasticola
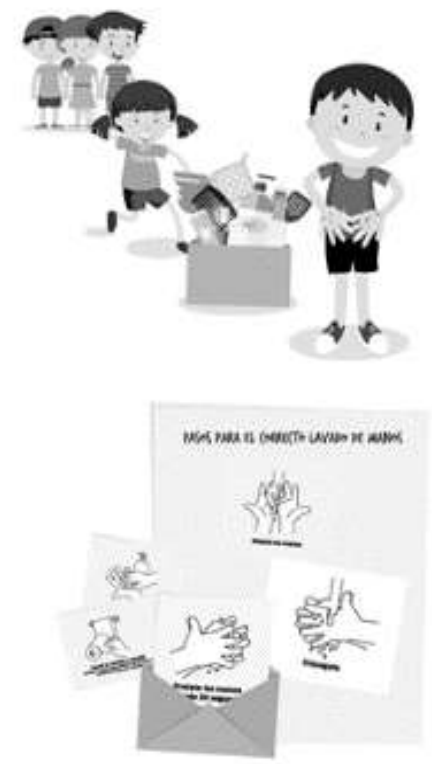

\section{TippyTap}

Se explica y realiza la construcción de un TippyTap a partir de estructuras pre-armadas. Se reparten las tareas entre los niños: colocar el palo horizontal, enhebrar el jabón y atarlo al palo, atar la base de la botella, llenar las botellas con agua y colgarlas del TippyTap, y mostrar su uso para el lavado de manos. Al finalizar, se reflexiona sobre cómo se construyó y cómo reemplazar los materiales si escasean.

Figura 1. Actividades del evento.

\section{Instrumentos}

Para la evaluación del evento se diseñó una encuesta para ponderar el conocimiento en los cuatro contenidos vinculados al lavado de manos

\section{Objetivo}

Aprender cuáles son los materiales necesarios para lavarse las manos

\section{Materiales}

Palos de madera, soga, botella de plástico, jabón, agua

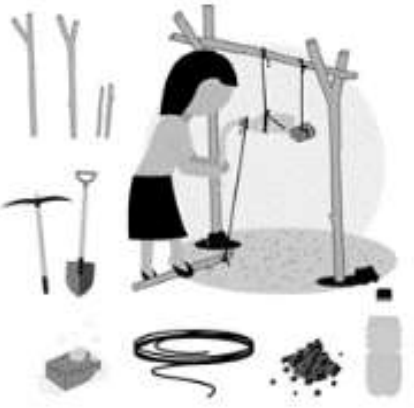
encuesta incluía cuatro preguntas, una pregunta por cada contenido.

La primera pregunta indagaba acerca de los que la intervención buscaba modificar. Cada 
pasos necesarios para un correcto lavado de manos y el orden en el cual deben realizarse. Para ello, los niños disponían de cinco renglones numerados para desarrollar sus respuestas, donde se consideró como correctas aquellas que contaron con todos los pasos en el orden correcto (1. Mojarse; 2. Enjabonarse; 3. Refregarse; 4. Enjuagarse; 5. Secarse). La segunda pregunta evaluaba el conocimiento acerca de los momentos en los cuales es necesario lavarse las manos, y constaba de un multiple choice con cinco opciones donde sólo una era correcta. Se tomó por válida la pregunta en los casos en los que se hubiere marcado la opción correcta únicamente ("Después de ir al baño"). La tercera pregunta hacía referencia a los motivos o razones por las cuales es necesario lavarse las manos y consistía en un multiple choice similar al anterior, con cinco opciones y sólo una correcta, por lo que se puntuó de igual modo, dándose por válida únicamente cuando estuviera marcada sólo la opción correcta ("Para no enfermarse"). Por último, la cuarta pregunta indagaba acerca de los elementos necesarios para el lavado de manos. Nuevamente era una pregunta con varios renglones para desarrollar, y fue considerada correcta si contenía como mínimo los dos elementos que deben utilizarse sí o sí (agua y jabón).

Las variables analizadas fueron la cantidad de correctos para Pasos, Momentos, Razones y Elementos. Las encuestas fueron llevadas hasta las escuelas que fueron invitadas a participar del evento por los coordinadores de cada localidad. Allí, fueron entregadas a las docentes de cuarto grado para que las repartieran entre los niños. Las encuestas fueron anónimas y no era posible identificar qué niño había respondido cada encuesta.

\section{Procedimiento}

En el Piloto 2016 (Puerto Iguazú), las escuelas fueron invitadas a participar del evento; las docentes de cada sala invitada a participar recibieron un documento con el detalle de las actividades y tuvieron oportunidad de realizar comentarios y sugerencias respecto de las mismas. Días antes del evento, las docentes se reunieron con los asistentes de los investigadores para trabajar sobre las actividades y fueron invitadas a llevar las actividades a cabo en conjunto con colaboradores de la investigación. Los niños fueron autorizados por sus padres para asistir al evento. Docentes y niños asistieron voluntariamente y participaron de las actividades. Al finalizar el evento se entregaron a los niños folletos, calcomanías y pulseras, como recordatorio de los contenidos trabajados. Un mes después las docentes administraron tanto a los niños que habían asistido al evento como a los que no, la encuesta sobre conocimiento del lavado de manos. En el piloto 2017 (cuatro localidades), se repitió el mismo procedimiento con la diferencia de que la encuesta se administró un mes antes (T1) y un mes después del evento (T2).

\section{Análisis de datos}

El análisis de datos fue el mismo para ambos pilotos (2016 y 2017): i) se hicieron correlaciones entre las variables para determinar si se detectaban comportamientos similares entre ellas y si debían analizarse separadamente; se consideró como indicador de identidad entre variables $r<=.75$; ii) se realizaron análisis de las frecuencias y porcentajes de correctos para cada variable; iii) se analizó la dificultad de la encuesta a fin de determinar la potencial existencia de efecto techo o piso en la prueba.

Para analizar los datos del piloto 2016, se compararon las frecuencias de respuestas correctas para cada variable que obtuvieron los niños que asistieron al evento con las de los niños que no asistieron al mismo. Para ello, se utilizó la prueba de chi cuadrado. Sin embargo, para analizar los datos del piloto 2017, se realizaron dos análisis. Primero, se ejecutó un análisis del conocimiento sobre el lavado de manos previo a la intervención para determinar cuál era el conocimiento de los niños antes del evento en estas localidades. Para ello se observaron las frecuencias de correctos para cada variable y se analizó si existían diferencias significativas entre ellas utilizando el estadístico de McNemar, dado que se trataba de muestras relacionadas.

En segundo lugar, se realizaron análisis comparando los puntajes en T1 y T2 para determinar si existían diferencias significativas entre ambos momentos. Para ello se utilizó el estadístico de ji cuadrado (para muestras independientes), dado que aunque es probable que los niños que hayan respondido la encuesta en T1 sean los mismos que en T2, no es posible tener la certeza de ello y, además, las encuestas eran 
Hermida, M. J., Ramírez, V. A., Goizueta, C. y Periago, M. V./ RACC, 2019, Vol. 11, №3, 33-45

anónimas por lo que no es posible determinar si un dato en T1 y un dato en T2 corresponden al mismo niño.

Por último, en aquellas variables en las que se observó una tendencia de la intervención a generar mejoras, se analizó si esa tendencia se observaba en todas las localidades o en alguna en particular. Para ello se realizaron los mismos análisis (ji cuadrado comparando la cantidad de correctos en T1 vs la cantidad de correctos en T2) para cada localidad.

Los análisis se realizaron utilizando la versión 15 del software SPSS.

\section{Aspectos éticos}

Las autoridades educativas de cada localidad autorizaron a los investigadores a trabajar con las escuelas para completar las encuestas y promover la asistencia de los niños al evento. En Pampa del Indio se obtuvo la autorización de la Sub Regional Educativa del Departamento Libertador General San Martín - Región Norte - Chaco del Ministerio de Educación, Cultura, Ciencia y Tecnología. En Clorinda se obtuvo la autorización de la Delegada Zonal del Ministerio de Cultura y Educación de la Provincia de Formosa. En Tartagal se hicieron eventos en cada escuela y se obtuvo autorización de las Directoras de las mismas. En Puerto Iguazú se obtuvo autorización de la Supervisora Escolar del Consejo de Educación del Ministerio de Cultura, Educación, Ciencia y Tecnología de Misiones, tanto para el piloto 2016 como para el piloto 2017. Los padres autorizaron a los niños a asistir al evento. Además, se realizaron reuniones con las directoras y docentes de las escuelas para solicitar su colaboración en la administración de las encuestas y la recolección de las autorizaciones de los padres. Las encuestas, administradas por las docentes, fueron anónimas y se realizaron como parte de una actividad escolar. Los niños fueron informados de los objetivos de la investigación y prestaron su asentimiento para responderlas.

\section{Resultados}

\section{Primer estudio piloto (Puerto Iguazú, 2016)}

En primer lugar, no se hallaron correlaciones significativas entre ninguna de las cuatro variables analizadas, por lo que cada variable se analizó por separado. Tampoco se detectó efecto techo en ninguna de las variables. Sin embargo, en cuanto a las razones para lavarse las manos, se observa un efecto piso, es decir, que la evaluación de esta variable pudo haber sido demasiado dificultosa para este grupo de niños.

Los resultados de este estudio piloto indicaron que los niños que asistieron no se diferencian de los que no asistieron con respecto a su conocimiento sobre los elementos necesarios para el lavado de manos $\left[\mathrm{x}^{2}(1,141)=.534 ; p=.465\right]$. Sin embargo, se observa que los niños que asistieron al evento tienen puntajes significativamente mayores a los de los niños que no asistieron en las variables Pasos $\left[\mathrm{X}^{2}(1,141)=15.152 ; p<.001\right]$, Momentos $\left[\mathrm{X}^{2}(1,141)=5.871 ; p=.015\right]$ y Razones para el lavado $\left[\mathrm{X}^{2}(1,141)=5.011 ; p=.025\right]$ (Figura 2). Destacablemente, la variable Razones tiene, incluso en aquellos niños que asistieron al evento, puntajes muy bajos.

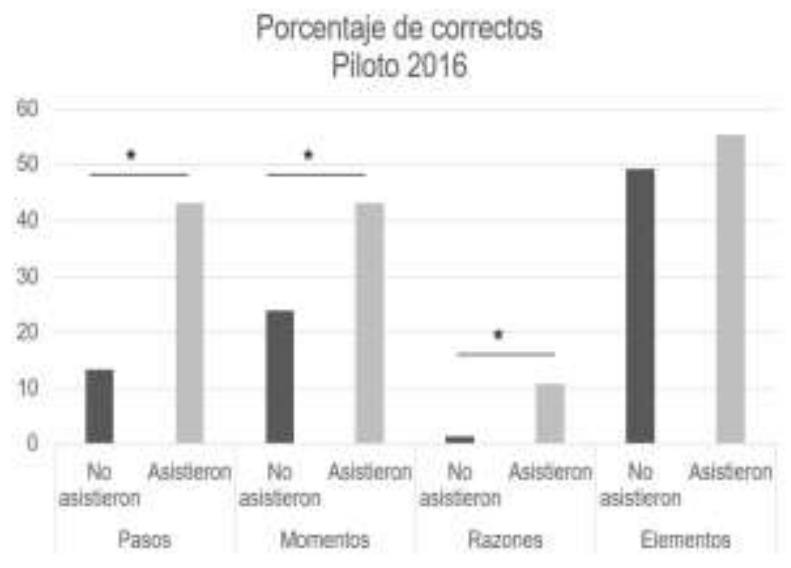

Figura 2. Porcentaje de respuestas correctas para los niños que asistieron y no asistieron al Piloto 2016, en cada variable analizada.

En síntesis, los resultados del estudio piloto de 2016 sugieren que los niños que asistieron al evento mostraron un mayor conocimiento sobre los pasos para un correcto lavado de manos, los momentos y las razones para lavarse las manos.

Sin embargo, estas diferencias en los conocimientos podrían existir en forma previa al evento y no ser producto del mismo. Para refinar la evaluación del impacto del evento, en el año 2017 se planificó un estudio de mayor envergadura, involucrando a otras localidades e incluyendo una evaluación previa y posterior al evento.

Además, para el evento 2017, y en función de la experiencia piloto, se modificaron aquellas actividades que habían resultado demasiado 
Hermida, M. J., Ramírez, V. A., Goizueta, C. y Periago, M. V./ RACC, 2019, Vol. 11, №3, 33-45

breves y se reformuló la pregunta de la encuesta sobre razones, por haber exhibido demasiada complejidad para los niños.

Segundo estudio piloto (Múltiples localidades, 2017)

En primer lugar, ninguna de las variables correlacionó entre sí, por lo que se procedió a analizar cada variable por separado.

En la Tabla 1 se presenta el tamaño muestral, la cantidad de correctos y el porcentaje de respuestas correctas para cada localidad en T1 y T2.

Tabla 1.

Tamaño muestral, cantidad de correctos y porcentaje de respuestas correctas para cada localidad T1 y en T2

\begin{tabular}{|c|c|c|c|c|c|c|c|c|c|c|c|c|c|}
\hline & & \multicolumn{3}{|c|}{ Pasos } & \multicolumn{3}{|c|}{ Momentos } & \multicolumn{3}{|c|}{ Razones } & \multicolumn{3}{|c|}{ Elementos } \\
\hline & Localidad & $n$ & Correctos & $\%$ & $n$ & Correctos & $\%$ & $n$ & Correctos & $\%$ & $n$ & Correctos & $\%$ \\
\hline \multirow[t]{5}{*}{ Pre } & Tartagal & 135 & 46 & 34.07 & 137 & 49 & 35.77 & 132 & 93 & 70.45 & 118 & 111 & 94.07 \\
\hline & Clorinda & 209 & 82 & 39.23 & 211 & 101 & 48.87 & 208 & 164 & 78.85 & 209 & 202 & 96.65 \\
\hline & $\begin{array}{l}\text { Iguazú } \\
\text { Pampa del }\end{array}$ & 281 & 48 & 17.08 & 290 & 77 & 26.55 & 286 & 180 & 62.94 & 271 & 241 & 88.93 \\
\hline & Indio & 60 & 5 & 8.33 & 61 & 29 & 47.54 & 60 & 36 & 60.00 & 55 & 54 & 98.18 \\
\hline & Total & 685 & 181 & 26.42 & 699 & 256 & 36.62 & 686 & 473 & 68.95 & 653 & 608 & 93.11 \\
\hline \multirow[t]{5}{*}{ Post } & Tartagal & 61 & 43 & 70.49 & 65 & 6 & 9.23 & 56 & 36 & 64.29 & 53 & 48 & 90.57 \\
\hline & Clorinda & 86 & 55 & 63.95 & 90 & 47 & 52.22 & 89 & 57 & 64.04 & 85 & 84 & 98.82 \\
\hline & $\begin{array}{l}\text { Iguazú } \\
\text { Pampa del }\end{array}$ & 308 & 87 & 28.25 & 323 & 122 & 37.77 & 321 & 220 & 68.54 & 291 & 260 & 89.35 \\
\hline & Indio & 91 & 26 & 28.57 & 94 & 31 & 32.98 & 95 & 45 & 47.37 & 84 & 83 & 98.81 \\
\hline & Total & 546 & 211 & 38.64 & 572 & 206 & 36.01 & 561 & 358 & 63.81 & 513 & 475 & 92.59 \\
\hline
\end{tabular}

La pregunta sobre Elementos fue respondida correctamente por más del $93 \%$ de los niños en T1. Esto indica que la pregunta no fue sensible para esta población, dado que los niños que participaron del estudio conocían que los elementos necesarios para el lavado de manos son agua y jabón.

El resto de las variables presentaron una dificultad adecuada, salvo la variable Pasos en la localidad Pampa del Indio, donde sólo un $8 \%$ de los niños pudieron describir correctamente los pasos para el lavado de manos. Esto indica que esta pregunta pudo haber resultado muy difícil para los niños de esta localidad.

\section{Análisis de T1}

El análisis de las frecuencias de respuestas en T1 indicó que, de los cuatro temas abordados, el más conocido por los niños antes del evento era Elementos (el 93\% de los niños sabía cuáles son los elementos necesarios para lavarse las manos), seguido por Razones (69\%), Momentos (37\%) y finalmente Pasos (sólo conocidos por el $26 \%$ de los niños). Las diferencias en la proporción de correctos entre las cuatro variables son significativas (Tabla 2).

Tabla 2.

Comparación de frecuencias de respuestas correctas en T1 (Prueba de McNemar).

\begin{tabular}{llll} 
& $n$ & $X^{2}$ & $p$ \\
\hline Pasos vs Momentos & 681 & 15.27 & .00 \\
Pasos vs Razones & 669 & 206.28 & .00 \\
Pasos vs Elementos & 644 & 412.19 & .00 \\
Momentos vs Razones & 685 & 181.11 & .00 \\
Momentos vs Elementos & 652 & 336.87 & .00 \\
Razones vs Elementos & 644 & 103.74 & .00 \\
\hline
\end{tabular}

\section{Comparación de T1 vs T2}

Si comparamos los puntajes obtenidos en T1 y T2 para cada variable, se observan diferencias significativas entre ambos momentos únicamente en la variable Pasos (Figura 3). Los resultados de las tablas de contingencia indican que la proporción de respuestas correctas a esta pregunta es significativamente mayor después de la intervención $\left[X^{2}(1,1231)=20.91, p<.001\right]$ : la 
Hermida, M. J., Ramírez, V. A., Goizueta, C. y Periago, M. V./ RACC, 2019, Vol. 11, №3, 33-45

proporción de respuestas correctas fue de .264 en T1 y de .386 en T2 (Figura 3). No se observan diferencias significativas en ninguno de los otros tres aspectos evaluados: Momentos $\left[X^{2}(1,1271)=\right.$ $.05, p=.86]$, Razones $\left[\mathrm{X}^{2}(1,1247)=3.662, p=.06\right]$ y Elementos $\left[X^{2}(1,1166)=.116, p=.73\right]$.

\section{Análisis de Pasos en T1 vs Pasos en T2, según localidad}

Para determinar si la diferencia observada en Pasos se verifica en todas las localidades, se compararon los correctos en la variable Pasos para cada localidad por separado. El aumento de respuestas correctas en T2 en la variable Pasos se verifica en todas las localidades. Si bien en Tartagal $\left[X^{2}(1,196)=22.48, p<.001\right]$ y en Clorinda $\left[X^{2}\right.$ $(1,295)=14.97, p<.001]$ las diferencias son de mayor magnitud, en Puerto Iguazú $\left[X^{2}(1,589)=\right.$ $10.37, p=.001]$ y en Pampa del Indio $\left[X^{2}(1,151)=\right.$ $9.58, p=.003$ ] las diferencias también son significativas.

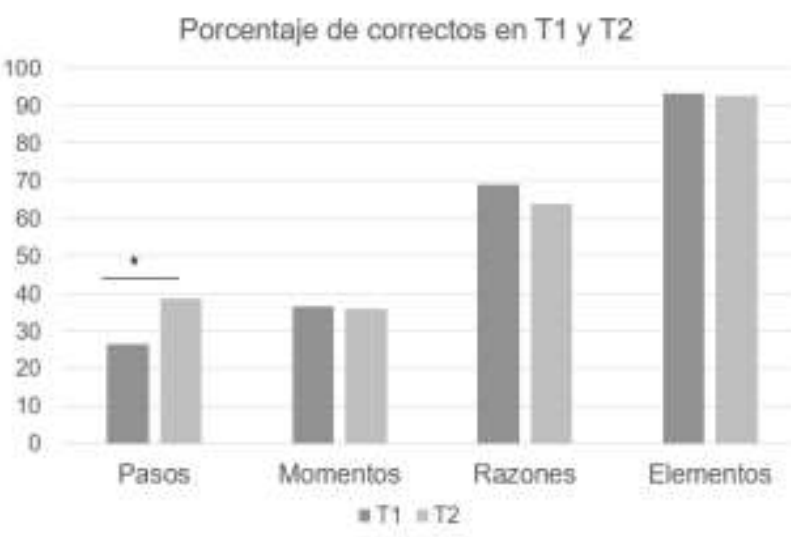

Figura 3. Porcentaje de respuestas correctas para cada aspecto del lavado de manos, en T1 y en T2 de los niños que asistieron al evento en el Piloto 2017. * $p<.001$

\section{Discusión}

El lavado de manos es un hábito que previene a muy bajo costo una gran cantidad de enfermedades (Curtis \& Cairncross, 2003; Garn et al., 2016; Grimes et al., 2016; Larson, 1988; Rabie \& Curtis, 2006; Wu et al., 2016); sin embargo, sólo una de cada cinco personas se lava las manos después de ir al baño (Freeman et al., 2014). Distintas intervenciones se han implementado para promover este hábito a nivel mundial (Naikoba \& Hayward, 2001). En Argentina, las intervenciones reportadas (Rodríguez et al., 2015; Rosenthal et al., 2004, 2005) involucran a adultos, pero no se han reportado intervenciones locales para promover este hábito en niños. Es fundamental encarar acciones para generar el hábito en niños, ya que estos son más vulnerables a muchas de las enfermedades que pueden prevenirse con el lavado de manos (Rabie \& Curtis, 2006), porque es más fácil modificar hábitos en niños (Bandura, 2004) y porque los hábitos generados en la infancia tienen mayor probabilidad de perdurar a lo largo de la vida (Rothman et al., 2015). Asimismo, estudios sugieren que los niños son buenos agentes de cambio específicamente en conductas de higiene de manos (O'Reilly et al., 2008). Para poder generar un cambio de hábitos, una condición necesaria es tener información acerca del hábito saludable y sus beneficios. En este sentido, adquirir conocimiento sobre el lavado de manos es un requisito previo. Por último, es especialmente importante trabajar en la adquisición de estos hábitos en poblaciones de bajo nivel económico social no-urbana, ya que tienen menos acceso a la salud (Gamboa et al., 1996; Milano et al., 2007). El presente trabajo describe dos estudios piloto locales que buscan cubrir estas áreas de vacancia: presenta el diseño, la ejecución y la evaluación de una intervención de bajo costo para promover el conocimiento sobre el lavado de manos en niños de 10 años, de localidades pobres no-urbanas de Argentina.

El análisis de los resultados del piloto en 2016 indicó que un mes después del evento, los niños que participaron del mismo obtuvieron en la encuesta una proporción de respuestas correctas significativamente mayor que los niños que no asistieron al evento, en tres variables: Pasos, Momentos y Razones. En la misma línea, los resultados del piloto 2017 indicaron que, un mes después del evento, los niños mostraron un aumento significativo de la cantidad de respuestas correctas en la variable Pasos. Este efecto se mantuvo considerando cada localidad por separado. Estos resultados indican que esta intervención de corta duración (un día, 3 a 4 horas) y de bajo costo tiene potencial para promover el conocimiento sobre los pasos que deben seguirse para un correcto lavado de manos. Si bien no podemos afirmar que tenga impacto, dado que no pudimos obtener datos sobre el grupo control, los resultados muestran una tendencia de la intervención a favorecer el conocimiento sobre el cómo deben lavarse las manos. Este impacto es importante, dado para generar un cambio de hábitos, es un requisito necesario conocer los 
comportamientos saludables, así como sus beneficios (Bandura, 1977, 2004; McAlister et al., 2008; Fisher \& Fisher, 1992).

Estos resultados llevan a la pregunta de por qué se observa un efecto únicamente en esta variable y no en las otras. Una posible explicación indica que el efecto depende de los valores que tenía cada variable en el momento previo a la intervención. Es decir, que los aumentos se observan en aquellas variables que los niños desconocían más en forma previa al evento. Antes del evento, el 93\% los niños de 10 años de todas las localidades evaluadas sabían cuáles son los elementos necesarios para un buen lavado de manos y el $69 \%$ de ellos conocían las razones por las que hay que lavarse las manos, por lo que el margen de mejora pudo haber sido menor en estas variables. El evento tuvo un impacto significativo justamente en una de las variables menos conocidas por los niños: Pasos. Esto además concuerda con los resultados del piloto 2016, en donde los niños que habían participado del evento obtuvieron mejores desempeños en esta variable, comparados con los niños que no habían asistido. Sin embargo, la variable Momentos no tenía niveles altos en T1 y permaneció en los mismos niveles en T2. Esto pudo deberse a otras razones, por ejemplo, a que el juego utilizado para estimular Momentos fue poco eficaz en plasmar ese conocimiento. Si bien no fue posible evaluar el efecto producido por cada juego en particular, es posible que la eficacia para promover conocimiento varíe según el juego. Esta hipótesis también podría explicar por qué el conocimiento de las razones para lavarse las manos, que en el piloto 2016 había mejorado post-intervención, no cambia entre T1 y T2 en el estudio de 2017. Una posible explicación para esto es que el juego para explicar Razones se modificó entre el piloto de 2016 y el de 2017. Otra posible explicación es la mencionada anteriormente: que esta variable también tenía niveles altos al inicio por lo que hay menos margen para moverlos.

Un aspecto importante a considerar respecto del piloto 2017 es el efecto aprendizaje, es decir si la misma aplicación de la prueba dos veces podría producir un aumento en el conocimiento per se (Lemay, Bédard, Rouleau, \& Tremblay, 2004). Se intentó disminuir este efecto haciendo las versiones parafraseadas del cuestionario y en el piloto 2017, separando su administración dos meses. Pero por otra parte, si esta fuera la explicación deberían haberse observado mejoras en todas las variables (ya que todas fueron expuestas a un doble testeo) y las diferencias significativas sólo se concentran en la variable Pasos. Para descartar esta posible explicación se debería realizar el evento con un grupo control.

En futuros estudios se deberán profundizar preguntas relevantes para estas intervenciones. Por ejemplo, esta intervención fue de un único día. Dado que la evidencia indica que el sostenimiento de las intervenciones aumenta su efectividad (Naikoba \& Hayward, 2001), sería interesante probar si la repetición de la intervención aumenta su efecto. Además, desconocemos si la intervención podría tener otros efectos en niños de otras edades. Sería valioso extender estas intervenciones a un espectro etario más amplio. También es importante controlar si el tema del lavado de manos se trabajó en clase, para separar ese efecto del efecto de la intervención. Finalmente, queda pendiente el análisis de cuánto del aumento en el conocimiento se traduce en un cambio de hábito concreto (Demberere et al., 2016; Rabbi \& Dey, 2013; Tamilarasi et al., 2016). De acuerdo con la Teoría Cognitiva Social, diversos determinantes son necesarios para llegar a producir un cambio de comportamiento. El primero de ellos es el conocimiento, y nuestros resultados sugieren que éste es pasible de ser aumentado por medio de esta intervención. Sin embargo, el presente estudio no indagó otros determinantes de importancia tales como la autoeficacia percibida, las expectativas de resultados, o los facilitadores e impedimentos sociales y estructurales. Futuros estudios deberán indagar estos aspectos, así como medir en forma directa cambios la frecuencia del lavado de manos. Este tipo de cambio en acciones cotidianas es difícil de medir controladamente y la mayoría de los estudios lo hacen indirectamente a través de reportes (Naikoba \& Hayward, 2001), pero es sin duda una pregunta crucial para poder conocer en profundidad el impacto de este tipo de intervenciones.

Más allá de todas estas limitaciones, la contribución del presente trabajo es presentar la primera intervención reportada en Argentina para favorecer el lavado de manos en niños. Es además, una intervención corta y de bajo costo, posible de ser aplicada y replicada en contextos de bajo nivel económico social. $\mathrm{Y}$, si bien no es posible determinar su impacto debido a la falta de un grupo control, los resultados de ambos pilotos sugieren 
Hermida, M. J., Ramírez, V. A., Goizueta, C. y Periago, M. V./ RACC, 2019, Vol. 11, №3, 33-45

que podría ser una intervención efectiva para aumentar el conocimiento acerca de cuáles son los pasos que deben seguirse para un correcto lavado de manos.

Se ha sugerido que un adecuado entendimiento de las dificultades para incorporar hábitos de higiene, así como las posibilidades de diseñar una intervención efectiva para promoverlos, dependen de un trabajo interdisciplinario (Gurses et al., 2010). La intervención del presente trabajo es transversal a diversas áreas. En primer lugar, fue diseñada interdisciplinariamente, entre especialistas en diagnóstico y prevención de transmisión de parásitos y especialistas en aprendizaje infantil. Además, articuló su implementación y evaluación con el ámbito educativo. En este sentido, el presente trabajo muestra un ejemplo de cómo es posible iniciar un camino que conduzca de la ciencia a la política pública. Ante un problema planteado y analizado principalmente desde el ámbito de la salud pública, se diseñó interdisciplinariamente un abordaje posible y se articuló con instituciones del ámbito educativo para la ejecución y evaluación. Este trabajo es un ejemplo, entre otros, de cómo iniciar intervenciones en pequeña escala (que a largo plazo podrían constituir políticas públicas), basadas en evidencia y articuladas con otros actores sociales como el sistema educativo.

\section{Agradecimientos}

Los autores agradecen al equipo de Mundo Sano que participó en el armado y acompañamiento de los eventos realizados: Diego Cruz (Tartagal), Evangelina Aldama (Clorinda), Cintia Delgado (Pampa del Indio) y Sofía Pereyra (Buenos Aires). También agradecemos al equipo multidisciplinario que brindó asesoramiento: la Profesora Nacional de Educación Física Gabriela Eliana Paiva y el diseñador Federico Fontán.

Este trabajo fue subsidiado por la Fundación Mundo Sano.

\section{Referencias}

Bandura, A. (1977). Self-efficacy: toward a unifying theory of behavioral change. Psychological review, 84(2), 191-215. doi: 10.1037/0033-295X.84.2.191.

Bandura, A. (2004). Health promotion by social cognitive means. Health Education \& Behavior, 31(2), 143-164. doi: $10.1177 / 1090198104263660$.

Basdevant, A., Boute, D., \& Borys, J. M. (1999). Who should be educated? Education strategies: could children educate their parents?. International Journal of Obesity, 23(S4), S10-S14. doi: 10.1038/sj.ijo.0800914.

Betts, J., Mckay, J., Maruff, P., \& Anderson, V. (2006). The development of sustained attention in children: The effect of age and task load. Child Neuropsychology, 12(3), 205-221. doi: 10.1080/09297040500488522.

Borra, S. T., Kelly, L., Shirreffs, M. B., Neville, K., \& Geiger, C. J. (2003). Developing health messages: qualitative studies with children, parents, and teachers help identify communications opportunities for healthful lifestyles and the prevention of obesity. Journal of the American Dietetic Association, 103(6), 721-728. doi: 10.1053/jada.2003.50140.

Boyce, J. M., \& Pittet, D. (2002). Guideline for hand hygiene in health-care settings: recommendations of the Healthcare Infection Control Practices Advisory Committee and the HICPAC/SHEA/APIC/IDSA Hand Hygiene Task Force. Infection Control \& Hospital Epidemiology, 23(S12), S3-S40.

Cairncross, S., Bartram, J., Cumming, O., \& Brocklehurst, C. (2010). Hygiene, sanitation, and water: what needs to be done?. PLoS Medicine, 7(11), e1000365. doi: 10.1371/journal.pmed.1000365.

Chin, Y. T., Lim, Y. A. L., Chong, C. W., Teh, C. S. J., Yap, I. K. S., Lee, S. C. ... Chua, K. H. (2016). Prevalence and risk factors of intestinal parasitism among two indigenous sub-ethnic groups in Peninsular Malaysia. Infectious Diseases of Poverty, 5(1), 77. doi: 10.1186/s40249-016-0168-z.

Curtis, V., \& Cairncross, S. (2003). Effect of washing hands with soap on diarrhea risk in the community: a systematic review. The Lancet Infectious Diseases, 3(5), 275-281. doi: 10.1016/S1473-3099(03)00606-6.

Demberere, T., Chidziya, T., Ncozana, T., \& Manyeruke, N. (2016). Knowledge and practices regarding water, sanitation and hygiene (WASH) among mothers of under-fives in Mawabeni, Umzingwane District of Zimbabwe. Physics and Chemistry of the Earth, Parts $A / B / C$, 92, 119-124. doi: 10.1016/j.pce.2015.09.013.

Diamond, A. (2013). Executive functions. Annual Review of Psychology, 64, 135-168. doi: 10.1146/annurev-psych113011-143750.

Dib, J., Oquilla, J., Lazarte, S. G., \& Gonzalez, S. N. (2012). Parasitic prevalence in a suburban school of Famaillá, Tucumán, Argentina. ISRN Microbiology, 2012, 560376. doi: 10.5402/2012/560376

Ejemot-Nwadiaro, R. I., Ehiri, J. E., Arikpo, D., Meremikwu, M. M., \& Critchley, J. A. (2015). Hand washing promotion for preventing diarrhoea. Cochrane Database of Systematic Reviews 2015, 9. doi: 10.1002/14651858.CD004265.pub3.

Fisher, J. D., \& Fisher, W. A. (1992). Changing AIDS risk behavior. Psychological Bulletin, 111(3), 455-474. doi: 10.1037/0033-2909.111.3.455

Freeman, M. C., Chard, A. N., Nikolay, B., Garn, J. V., Okoyo, C., Kihara, J. ... Mwandawiro, C. S. (2015). Associations between school-and household-level water, sanitation and hygiene conditions and soiltransmitted helminth infection among Kenyan school children. Parasites \& Vectors, 8(1), 412. doi: 
10.1186/s13071-015-1024-x.

Freeman, M. C., Stocks, M. E., Cumming, O., Jeandron, A., Higgins, J. P., Wolf, J. ... Curtis, V. (2014). Systematic review: hygiene and health: systematic review of handwashing practices worldwide and update of health effects. Tropical Medicine \& International Health, 19(8), 906-916. doi: 10.1111/tmi.12339.

Gamboa, M. I., Basualto Farjat, J. Á., Kozubsky, L., Costas, M. E., Cueto Rúa, E., \& Lahitte, H. B. (1996). Enteroparasitosis en dos poblaciones periurbanas de La Plata, Argentina. Boletín Chileno de Parasitología, 51(1/2), 37-41.

Gamboa, M. I., Navone, G. T., Orden, A. B., Torres, M. F., Castro, L. E., \& Oyhenart, E. E. (2011). Socioenvironmental conditions, intestinal parasitic infections and nutritional status in children from a suburban neighborhood of La Plata, Argentina. Acta Tropica, 118(3), 184-189.

10.1016/j.actatropica.2009.06.015.

Garn, J. V., Mwandawiro, C. S., Nikolay, B., Drews-Botsch, C. D., Kihara, J. H., Brooker, S. J. ... \& Freeman, M. C. (2016). Ascaris lumbricoides infection following schoolbased deworming in western Kenya: assessing the role of pupils' school and home water, sanitation, and hygiene exposures. The American Journal of Tropical Medicine and Hygiene, 94(5), 1045-1054. doi: 10.4269/ajtmh.15-0362.

Global Handwashing Partnership (2017) Guía para planificadores. Disponible en: https://globalhandwashing.org/resources/ghdplanners-guide/?_sft_content=partnership-documents.

Global Handwashing Partnership (2017) Global handwashing day. Recuperado de: https://globalhandwashing.org/global-handwashingday/

Gortmaker, S. L., Peterson, K., Wiecha, J., Sobol, A. M., Dixit, S., Fox, M. K., \& Laird, N. (1999). Reducing obesity via a school-based interdisciplinary intervention among youth: Planet Health. Archives of Pediatrics \& Adolescent Medicine, 153(4), 409-418. doi: 10.1001/archpedi.153.4.409.

Grimes, J. E., Tadesse, G., Mekete, K., Wuletaw, Y., Gebretsadik, A., French, M. D. ... \& Templeton, M. R. (2016). School water, sanitation, and hygiene, soiltransmitted helminths, and schistosomes: national mapping in Ethiopia. PLoS Neglected Tropical Diseases, 10(3), e0004515. doi: 10.1371/journal.pntd.0004515.

Gurses, A. P., Marsteller, J. A., Ozok, A. A., Xiao, Y., Owens, S., \& Pronovost, P. J. (2010). Using an interdisciplinary approach to identify factors that affect clinicians' compliance with evidence-based guidelines. Critical Care Medicine, 38, S282-S291. doi: 10.1097/CCM.0b013e3181e69e02.

Heft, H., \& Chawla, L. (2006). Children as agents in sustainable development: the ecology of competence. En C. Spencer, \& M. Blades (Eds.), Children and their environments: Learning, using and designing spaces (pp. 199-216). Cambridge: Cambridge University Press
Hermida, M. J., Segretin, M. S., Prats, L. M., Fracchia, C. S., Colombo, J. A., \& Lipina, S. J. (2015). Cognitive neuroscience, developmental psychology, and education: Interdisciplinary development of an intervention for low socioeconomic status kindergarten children. Trends in Neuroscience and Education, 4(12), 15-25. doi: 10.1016/j.tine.2015.03.003.

Instituto Nacional de Estadísticas y Censos, INDEC (2010). Anuario estadístico de la República Argentina. Buenos Aires: INDEC.

Larson, E. (1988). A causal link between handwashing and risk of infection? Examination of the evidence. Infection Control \& Hospital Epidemiology, 9(1), 28-36. doi: 10.1086/645729.

Larson, E. L., \& APIC Guidelines Committee. (1995). APIC guidelines for handwashing and hand antisepsis in health care settings. American Journal of Infection Control, 23(4), 251-269. doi: 10.1016/01966553(95)90070-5.

Lemay, S., Bédard, M. A., Rouleau, I., \& Tremblay, P. L. (2004). Practice effect and test-retest reliability of attentional and executive tests in middle-aged to elderly subjects. The Clinical Neuropsychologist, 18(2), 284-302. doi: 10.1080/13854040490501718.

Mahmud, M. A., Spigt, M., Bezabih, A. M., Pavon, I. L., Dinant, G. J., \& Velasco, R. B. (2015). Efficacy of handwashing with soap and nail clipping on intestinal parasitic infections in school-aged children: a factorial cluster randomized controlled trial. PLOS Medicine, 12(6), e1001837. doi: 10.1371/journal.pmed.1001837.

Manz, K. M., Clowes, P., Kroidl, I., Kowuor, D. O., Geldmacher, C., Ntinginya, N. E., ... Saathoff, E. (2017). Trichuris trichiura infection and its relation to environmental factors in Mbeya region, Tanzania: A cross-sectional, population-based study. PloS One, 12(4), e0175137. doi: 10.1371/journal.pone.0175137

McAlister, A. L., Perry, C. L., \& Parcel, G. S. (2008). How individuals, environments, and health behaviors interact: Social cognitive theory. En K. Glanz, B. K. Rimer, \& K. Viswanath (Eds.), Health behavior and health education: Theory, research, and practice (pp. 169-188). San Francisco, CA, US: Jossey-Bass.

Milano, A. M., Oscherov, E. B., Palladino, A. C., \& Bar, A. R. (2007). Children enteroparasitosis in North East Argentine urban area. Medicina, 67(3), 238-242.

Mitchell, I. K., Ling, C., Krusekopf, C., \& Kerr, S. (2015). Pathways toward whole community transformation: a case study on the role of school engagement and environmental education. Environment, Development and Sustainability, 17(2), 279-298. doi: 10.1007/s10668-014-9587-9.

Mitchell, R. E., Fraser, A. M., \& Bearon, L. B. (2007). Preventing food-borne illness in food service establishments: Broadening the framework for intervention and research on safe food handling behaviors. International Journal of Environmental Health Research, 17(1), 9-24. doi: 10.1080/09603120601124371.

Naikoba, S., \& Hayward, A. (2001). The effectiveness of interventions aimed at increasing handwashing in 
Hermida, M. J., Ramírez, V. A., Goizueta, C. y Periago, M. V./ RACC, 2019, Vol. 11, №3, 33-45

healthcare workers-a systematic review. Journal of Hospital Infection, 47(3), 173-180. doi: 10.1053/jhin.2000.0882.

O'Reilly, C. E., Freeman, M. C., Ravani, M., Migele, J., Mwaki, A., Ayalo, M. ... Quick, R. (2008). The impact of a school-based safe water and hygiene programme on knowledge and practices of students and their parents: Nyanza Province, western Kenya, 2006. Epidemiology \& Infection, 136(1), 80-91. doi: $10.1017 /$ S0950268807008060.

Percy-Smith, B., \& Burns, D. (2013). Exploring the role of children and young people as agents of change in sustainable community development. Local Environment, 18(3), 323-339. doi: 10.1080/13549839.2012.729565.

Pittet, D., Allegranzi, B., Boyce, J., \& World Health Organization World Alliance for Patient Safety First Global Patient Safety Challenge Core Group of Experts. (2009). The World Health Organization guidelines on hand hygiene in health care and their consensus recommendations. Infection Control \& Hospital Epidemiology, 30(7), 611-622. doi: 10.1086/600379.

Rabbi, S. E., \& Dey, N. C. (2013). Exploring the gap between hand washing knowledge and practices in Bangladesh: a cross-sectional comparative study. BMC Public Health, 13(1), 89. doi: 10.1186/1471-245813-89

Rabie, T., \& Curtis, V. (2006). Handwashing and risk of respiratory infections: a quantitative systematic review. Tropical Medicine \& International Health, 11(3), 258267. doi: 10.1111/j.1365-3156.2006.01568.x.

Rodríguez, V., Giuffre, C., Villa, S., Almada, G., PrasopaPlaizier, N., Gogna, M., ... Vidal, A. (2015). A multimodal intervention to improve hand hygiene in ICUs in Buenos Aires, Argentina: a stepped wedge trial. International Journal for Quality in Health Care, 27(5), 405-411. doi: 10.1093/intqhc/mzv065

Rosenthal, V. D., Guzman, S., \& Safdar, N. (2004). Effect of education and performance feedback on rates of catheter-associated urinary tract infection in intensive care units in Argentina. Infection Control \& Hospital Epidemiology, 25(1), 47-50. doi: 10.1086/502291.

Rosenthal, V. D., Guzman, S., \& Safdar, N. (2005). Reduction in nosocomial infection with improved hand

hygiene in intensive care units of a tertiary care hospital in Argentina. American Journal of Infection Control, 33(7), 392-397. doi: 10.1016/j.ajic.2004.08.009.

Rothman, A. J., Gollwitzer, P. M., Grant, A. M., Neal, D. T., Sheeran, P., \& Wood, W. (2015). Hale and hearty policies: How psychological science can create and maintain healthy habits. Perspectives on Psychological Science, 10(6), 701-705. doi: 10.1177/1745691615598515.

Steinmann, P., Zhou, X. N., Li, Y. L., Li, H. J., Chen, S. R., Yang, Z., ... Utzinger, J. (2007). Helminth infections and risk factor analysis among residents in Eryuan county, Yunnan province, China. Acta Tropica, 104(1), 38-51. doi: 10.1016/j.actatropica.2007.07.003.

Tamilarasi, R., Arunmozhi, R., Raja, V. K., \& Rajajeyakumar, M. (2016). A Study to Assess the Knowledge and Practice of Hand Washing among School Going Adolescents in Chennai. International Journal of Health Sciences and Research, 6(8),147155.

Walker, C. L. F., Rudan, I., Liu, L., Nair, H., Theodoratou, E., Bhutta, Z. A. ... Black, R. E. (2013). Global burden of childhood pneumonia and diarrhoea. The Lancet, 381(9875), 1405-1416. doi: 10.1016/S01406736(13)60222-6.

Whitby, M., Pessoa-Silva, C. L., McLaws, M. L., Allegranzi, B., Sax, H., Larson, E. ... Pittet, D. (2007). Behavioural considerations for hand hygiene practices: the basic building blocks. Journal of Hospital Infection, 65(1), 18. doi: 10.1016/j.jhin.2006.09.026.

Woldemicael, G. (2001). Diarrhoeal morbidity among young children in Eritrea: environmental and socioeconomic determinants. Journal of Health, Population and Nutrition 19(2), 83-90.

Wu, S., Ma, C., Yang, Z., Yang, P., Chu, Y., Zhang, H. ... Wang, Q. (2016). Hygiene behaviors associated with influenza-like illness among adults in Beijing, China: a large, population-based survey. PloS One, 11(2), e0148448. doi: 10.1371/journal.pone.0148448.

Zonta, M. L., Navone, G. T., \& Oyhenart, E. E. (2007). Parasitosis intestinales en niños de edad preescolar y escolar: situación actual en poblaciones urbanas, periurbanas y rurales en Brandsen, Buenos Aires, Argentina. Parasitología Latinoamericana, 62(1-2), 5460. doi: 10.4067/S0717-77122007000100009. 